\title{
Review of: "Genetic Heterogeneity in GJB2, COL4A3, ATP6V1B1 and EDNRB Variants Detected Among Hearing Impaired Families in Morocco"
}

\author{
Malika DAHMANI
}

Potential competing interests: The author(s) declared that no potential competing interests exist.

Thank you for working in this field. I have provided some suggestions which I hope you will find useful to strengthen your manuscript.

1. In the abstract, the genes are not italicized.

2. It is not clear how the selection of the patients included in the study was made

3. It is not clear how often the blood was collected for genetic analysis.

4. I suggest includ clinical carracteristics of patients with syndromic deafness as well as results in table.

5. In the discussion, the authors did not cite that c.1155dupC;p.(Ile386HisfsTer56) of ATP6V1B1 has also been identified in Algeria.

6. I suggest including in the manuscript data on the main treatment options . 